\title{
ARCO DE SCOTIA ¿UN PUENTE PARA EL INTERCAMBIO DE LOS FORAMINÍFEROS BENTÓNICOS ENTRE ANTÁRTICA Y SUDAMÉRICA?
}

\author{
SCOTIA ARC, A BRIDGE FOR ANTARCTIC AND SOUTH AMERICAN \\ BENTHIC FORAMINIFERA INTERCHANGE?
}

Tatiana Hromic ${ }^{1}$

\begin{abstract}
During the LAMPOS 2002 cruise, six marine sediment samples were collected in Scotia Arc area (Orkney Island, South Georgia, South Sandwich and Elephant Island) from 178 - 255 m water depth. In order to investigate benthic foraminifera the samples were analyzed. In total twenty four species were identified, belonging to 4 suborders, 18 families and 20 genera. Textulariina is the most abundant suborder (81,4\% speciemens and $58,3 \%$ species) in all the samples, except Isla Elefante. The main species were: Miliammina arenacea (23,1\%), Reophax pilulifer (13,5\%); Trochammina antartica $(12,7 \%)$; Bathysiphon sp. (10,7\%); Trochammina inflata (7,4\%) y Fursenkoina fusiformis (6,2\%). The Scotia Arc could be a bridge for 13 species. For Pseudobolivina antarctica, Portatrochammina antartica and Trochammina antartica present only in Antartica and for Cyclammina cancellata and Ammobaculites americanus, only present in South America, the Scotia Arc would be the biogeographic limit.
\end{abstract}

Key words: Foraminifera, benthonic, Scotia Arc, Antartic biodiversity, abundance.

\section{RESUMEN}

Durante el crucero LAMPOS- 2002, se recolectaron 6 muestras de sedimentos marinos en el Arco de Scotia (Islas Orcadas, Georgias del Sur, Sandwich del Sur e Isla Elefante) desde profundidades entre 178 y 255 m. Las muestras fueron analizadas para investigar la presencia de foraminíferos bentónicos. Se identificaron 24 especies clasificadas en 4 subórdenes, 18 familias y 20 géneros. El suborden con mayor representación fue Textulariina ( $81,4 \%$ de los ejemplares y el 58,3\% de las especies), en todas las muestras, excepto en Isla Elefante. Las especies más abundantes fueron: Miliammina arenacea

\footnotetext{
${ }^{1}$ Laboratorio de Micropaleontología, Instituto de la Patagonia, Universidad de Magallanes, Casilla 113-D, Punta Arenas, Chile.
} tatiana.hromic@umag.cl 
(23,1\%), Reophax pilulifer (13,5\%); Portatrochammina antartica (12,7\%); Bathysiphon sp. (10,7\%); Trochammina inflata $(7,4 \%)$ y Fursenkoina fusiformis (6,2\%). El Arco de Scotia actuaría como puente entre ambos continentes para 13 especies. Las especies Pseudobolivina antarctica, Portatrochammina antartica y Trochammina antartica son endémicas de la Antártica y para Cyclammina cancellata y Ammobaculites americanus, sólo presentes en Sudamérica, el arco de Scotia se constituiría en el límite biogeográfico.

Palabras clave: Foraminíferos, bentónicos, Arco de Scotia, Antártica, abundancia, diversidad.

\section{INTRODUCCIÓN}

El enfriamiento de la Antártica, en su migración hacia el Sur al desprenderse de Gondwana, la instalación de la corriente circumpolar y la profundización de los mares en su entorno, son factores que contribuyeron al aislamiento y diferenciación de las comunidades biológicas entre este continente y Sudamérica. Sin embargo, el hecho de que ambos continentes estuvieran unidos en el pasado y de que actualmente son los más próximos, ha motivado a diversos investigadores a preguntarse si existe alguna relación entre la fauna de Antártica y Sudamérica (Arntz, 2003 In Arntz \& Brey, 2003). La presencia de numerosas islas entre ambos continentes sugiere algún tipo de conexión y una posibilidad de intercambio similar al gran intercambio Pleistocénico, sucedido entre América del norte y del Sur, al menos en relación a la fauna marina. En este escenario, el Arco de Scotia jugaría un rol importante como corredor biológico entre Antártica y Sudamérica. El Arco de Scotia es una cordillera submarina, derivada de los Andes sudamericanos, que se continúan en los Antartandes y que forman un gran arco que rodea la placa de Scotia por el oriente. Varias de sus cumbre emergen formando grupos de islas muy conocidas como las Georgias del Sur, Sandwich del Sur, Orcadas del Sur y Shetland del Sur, y dan paso a un sistema de islas, que perfectamente podrían servir de puente para la fauna entre Sudamérica y Antártica (Arntz op.cit.).

Un grupo importante de la fauna marina son los foraminíferos bentónicos (Rhizopoda: Foraminiferida) (Adl et al. 2005) y, sin duda, para ellos también el Arco de Scotia podría servir como medio de comunicación entre ambos continentes. Algunos investigadores señalan que en las costas sudamericanas australes del Pacífico, hay un predominio de foraminíferos con caparazón calcáreo, rotalínidos principalmente (Ishman \& Martínez, 1995; Zapata \& Moyano, 1997; Hromic, 1996, 1999, 2001, 2005, 2007, 2009), mientras que en la Antártica, se observa una mayor presencia de organismos con caparazón arenáceo, textuláridos (Herb, 1971; Finger \& Lipps, 1981; Lena, 1975, 1980; Mackensen et al. 1990). A nivel específico, algunas especies como Cassidulina rossensis, descrita para Antártica (Kennett, 1967), se presenta con gran frecuencia y abundancia en la costa Pacífica (Hromic et al. 2006), mientras que otras como Miliammina arenacea se encuentra sólo en algunos puntos restringidos de la zonas de canales, internos (Zúñiga-Rival, 2006).

Los foraminíferos bentónicos de la zona austral Pacífica han sido estudiados por diversos autores en la últimas décadas (Marchant, 1993; Zapata \& Moyano, 1997; Violanti et al. 2000; Hromic et al. 2006); y los de Antártica por investigadores como, Lena $(1975,1980)$, Gaździcki \& Majewski (2003), Ishman \& Domack (1994) Majewski (2005) entre otros muchos, sin embargo, no se ha hecho a la fecha un estudio comparado de los foraminíferos entre ambos continentes con excepción de algunos intentos (Hromic, 2002; Hromic et al. 2012).

Escasos estudios se han hecho en las cercanías del Arco de Scotia, uno de ellos fue realizado por Herb (1971), en el paso Drake. Este autor encontró entre los arenáceos más abundantes: Reophax pilulifer, R. nodulosus, L. jeffreysii, Bathysiphon filiformis, Rhizammina indivisa, Saccammina sphaerica, Reophax nodulosus y Cyclammina pusilla y entre los calcáreos dominantes, Cibicides fletcheri, Discanomalina vermiculata y Cassidulina crassa. Otro estudio en las proximidades del Arco de Scotia, corresponde al realizado por Thompson (1978), en la Isla de los Estados $\left(\sim 54,5^{\circ} \mathrm{S} 64,5^{\circ} \mathrm{W}\right)$; en este se da a conocer una rica fauna formada principalmente por rotálidos con abundante presencia de Rosalina 


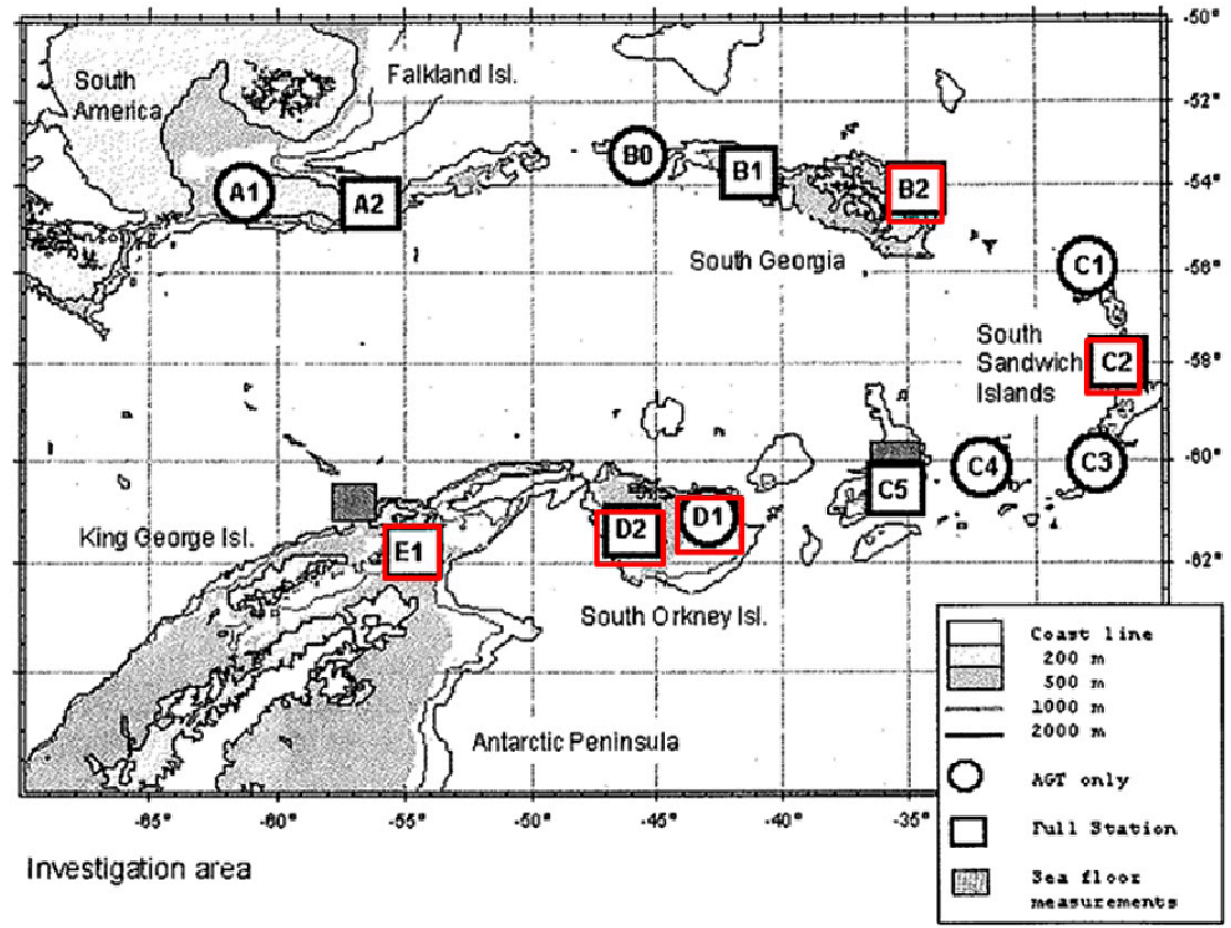

Fig. 1. Área de muestreo. Tomado de: Arntz \& Brey 2003. Se indica en color las áreas desde donde se obtuvieron las muestras.

globularis, Cibicides lobatulis, Elphidum spp.

El objetivo de este estudio es dar a conocer la biodiversidad de foraminiferos presentes en el Arco de Scotia y determinar cualitativamente las similitudes con la fauna del extremo sudaustral sudamericano y con la fauna de la península Antártica. Cabe hacer notar que es difícil establecer un símil entre ambas faunas debido a las diferentes técnicas de recolección y a las diversas profundidades desde las que se han recolectado las muestras. Por lo tanto, la comparación faunística sólo es posible desde un enfoque cualitativo.

\section{MATERIAL Y MÉTODOS}

El material estudiado proviene de 6 muestras de sedimento marino obtenidas por personal del buque Polarsten, durante el crucero "LAMPOS", en el arco de Scotia (54-62 ${ }^{\circ}$ S) (LAMPOS-2002) (Fig. 1) desde profundidades entre $178-255 \mathrm{~m}$. Las muestras se tomaron en las estaciones $\mathrm{N}^{\circ} 178$

Tabla 1: Caracterización de las muestras

\begin{tabular}{lccccc}
\hline Lugar & Estación/ muestra & Latitud S & Longitud W & Profundidad (m) & Instrumento \\
\hline $\begin{array}{l}\text { SE South } \\
\text { Islands (B2) }\end{array}$ & 178 & $54^{\circ} 25,46^{\prime}$ & $35^{\circ} 38,91^{\prime}$ & 277 & MUC \\
$\begin{array}{l}\text { Central South Sandwich } \\
\text { Islands (C2) }\end{array}$ & 211 & $57^{\circ} 35,58^{\prime}$ & $26^{\circ} 23,69^{\prime}$ & 1094 & MUC \\
$\begin{array}{l}\text { E South Orkney Islands } \\
\text { (D1) }\end{array}$ & 231 & $60^{\circ} 59,31^{\prime}$ & $43^{\circ} 27,80^{\prime}$ & 402 & AGT \\
$\begin{array}{l}\text { SW South Orkney Islands } \\
\text { (D2) }\end{array}$ & 243 & $61^{\circ} 11,42^{\prime}$ & $45^{\circ} 45,66^{\prime}$ & 305 & MUC \\
$\begin{array}{l}\text { SW South Orkney Islands } \\
\text { (D2) }\end{array}$ & 238 & $61^{\circ} 11,05^{\prime}$ & $45^{\circ} 76,45^{\prime}$ & 324 & AGT \\
S Elephant Island (E1) & 255 & $61^{\circ} 23,86^{\prime}$ & $55^{\circ} 23,80^{\prime}$ & 280 & MUC \\
\hline
\end{tabular}


(E Georgias del Sur), No 211 (Sandwich del Sur, central); $N^{\circ} 231$ y $N^{\circ} 238 ; N^{\circ} 243$ (Islas Orcadas este y sur-oeste respectivamente) y $\mathrm{N}^{\circ} 255$ (S Isla Elefante) (Tabla 1). Cuatro muestras fueron recolectadas con Multicorer (MUC) y 2 provienen de sedimentos recolectados con draga Agassiz. Los ejemplares recolectados se depositaron en bolsas que se refrigeraron hasta el momento de uso. Las

Tabla 2. Clasificación sistemática, abundancia relativa y frecuencia de los foraminíferos bentónicos recolectados en el Arco de Scotia.

\begin{tabular}{|c|c|c|c|c|c|}
\hline Suborden & Familia & Género & Especie & $\%$ & Frecuencia \\
\hline \multirow[t]{14}{*}{ TEXTULARIINA } & Lituolidae & Ammobaculites & (1) A. americanus & 2 & 1 \\
\hline & \multirow[t]{2}{*}{ Rhabdamminidae } & Bathysiphon & (2) Bathysiphon sp. & 10,7 & 5 \\
\hline & & Rhabdammina & (3) R. abyssorum Sars, 1869 & 0,9 & 1 \\
\hline & Cyclamminidae & Cyclammina & (4) C. cancellata Brady, 1879 & 0,1 & 2 \\
\hline & Ammodiscidae & Glomospira & (5) Glomospira sp. & 0,9 & 1 \\
\hline & Haplophragmoididae & Labrospira & $\begin{array}{l}\text { (6) L. kosterensis Höeglund } \\
1947\end{array}$ & 3,8 & 5 \\
\hline & Rzehakinidae & Miliammina & $\begin{array}{l}\text { (7) M. arenacea (Chapman, } \\
\text { 1916) }\end{array}$ & 23,1 & 6 \\
\hline & \multirow[t]{3}{*}{ Hormosinidae } & \multirow[t]{3}{*}{ Reophax } & (8) R. pilulifer Brady, 1884 & 13,5 & 5 \\
\hline & & & $\begin{array}{l}\text { (9) R. scorpiurus de Montfort, } \\
1808\end{array}$ & 0,5 & 4 \\
\hline & & & $\begin{array}{l}\text { (10) } R \text {. dentaliniformis } \\
\text { (Brady, 1884) }\end{array}$ & 3 & 5 \\
\hline & \multirow[t]{3}{*}{ Trochamminidae } & \multirow[t]{2}{*}{ Trochammina } & $\begin{array}{l}\text { (11) T. inflata (Montagu, } \\
\text { 1808) }\end{array}$ & 7,4 & 3 \\
\hline & & & $\begin{array}{l}\text { (12) T. malovensis Heron- } \\
\text { Allen \& Earland, } 1929\end{array}$ & 0,3 & 2 \\
\hline & & Portatrochammina & $\begin{array}{l}\text { (13) P. antarctica (Parr, } \\
\text { 1950) }\end{array}$ & 12,7 & 4 \\
\hline & Pseudobolivinidae & Pseudobolivina & $\begin{array}{l}\text { (14) P. antartica (Wiesner, } \\
\text { 1931) }\end{array}$ & 2,6 & 4 \\
\hline \multirow[t]{6}{*}{ ROTALIINA } & Uvigerinidae & Angulogerina & $\begin{array}{l}\text { (1) A. angulosa (Williamson, } \\
\text { 1858) }\end{array}$ & 2,9 & 3 \\
\hline & Buliminidae & Bulimina & $\begin{array}{l}\text { (2) B. aculeata d'Orbigny, } \\
1826\end{array}$ & 1,2 & 2 \\
\hline & Fursenkoinidae & Fursenkoina & $\begin{array}{l}\text { (3) F. fusiformes } \\
\text { (Williamson, 1858) }\end{array}$ & 6,2 & 5 \\
\hline & Trichohylidae & Bucella & (4) B. frigida (Cushman 1922) & 1,6 & 2 \\
\hline & Nonionidae & Pullenia & $\begin{array}{l}\text { (5) P. subcarinata (d'Orbigny, } \\
\text { 1839) }\end{array}$ & 4,4 & 6 \\
\hline & Cassidulinidae & Cassidulinoides & (6) C. parvus (Earland 1934) & 1,1 & 2 \\
\hline \multirow[t]{2}{*}{ MILIOLINA } & Cornuspiridae & Cornuspira & $\begin{array}{l}\text { (1) C. involvens (Reuss, } \\
\text { 1850) }\end{array}$ & 0,7 & 4 \\
\hline & Hauerinidae & Pyrgo & (2) Pyrgo sp. & 0,2 & 3 \\
\hline \multirow[t]{2}{*}{ LAGENINA } & \multirow[t]{2}{*}{ Lagenidae } & \multirow[t]{2}{*}{ Lagena } & $\begin{array}{l}\text { (1) Lagena dístoma Parker \& } \\
\text { Jones } 1864\end{array}$ & 0,1 & 2 \\
\hline & & & $\begin{array}{l}\text { (2) Lagena striata } \\
\text { (d'Orbigny, 1839) }\end{array}$ & 0,3 & 2 \\
\hline
\end{tabular}



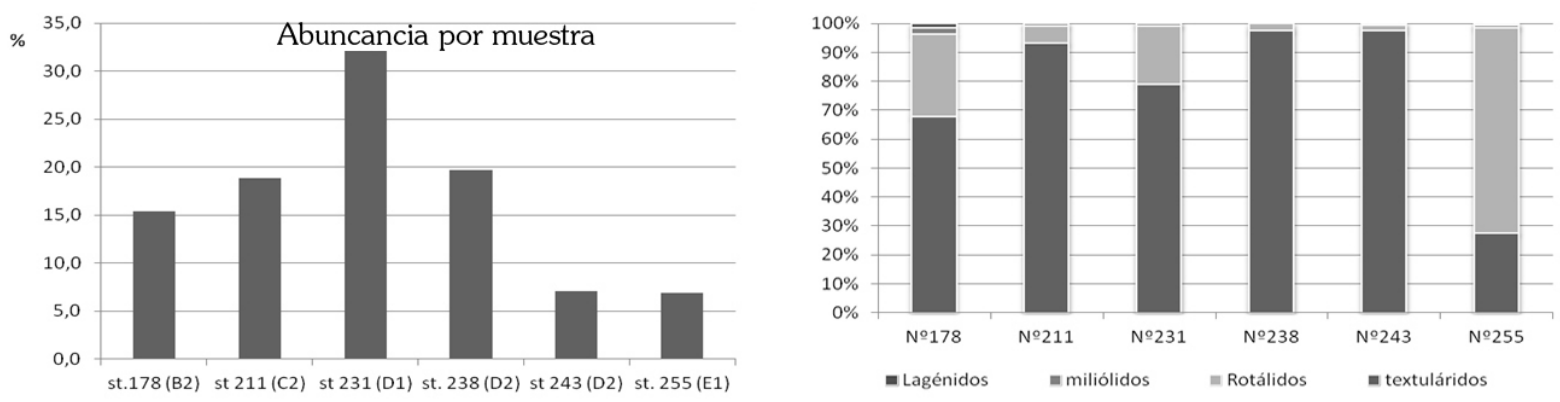

Fig. 2. Abundancia relativa de foraminíferos por muestra y distribución según suborden.

muestras fueron lavadas con un tamiz de malla de 63 micras, secadas a temperatura ambiental y pesadas. De cada muestra se trabajaron $50 \mathrm{~g}$ para estandarizar los resultados. Los foraminíferos fueron extraídos uno a uno bajo lupa binocular $y$ depositados en reglillas. Posteriormente se separaron en especies y se contaron (Boltovskoy, 1965). La identificación hasta el nivel genérico se basó en Loeblich \& Tappan (1988) y Decrouez (1989) y al nivel específico en el catálogo de Ellis \& Messina (1944 et seq.) y literatura del área (Brady, 1884; Barker, 1960; Lena, 1975; Boltoskoy et al. 1980; Heron Allen \& Earland, 1932).

Se aplicaron técnicas estadísticas univariadas para determinar diferencias/ semejanzas de la estructura comunitaria entre muestras. Para estudiar la composición faunística se calculó abundancia absoluta $(\mathrm{N})$ y relativa (\%); riqueza de taxa (S); equidad (J': índice de Pielou) y diversidad (H': índice de Shannon-Wiener) (Magurran, 1988). Para el análisis de conglomerados se utilizó el indice de Bray-Curtis y los datos se muestran en un dendrograma. La significancia estadística de los grupos formados se evaluó con la rutina
SIMPROF (similarity profile) al 95\% de confianza, utilizando como hipótesis nula que los conjuntos de foraminíferos bentónicos no diferían entre sí y como hipótesis a validar que las asociaciones de foraminíferos bentónicos en las distintas muestras son diferentes. Los cálculos se efectuaron con el programa PRIMER 6.0 (Clarke \& Gorley, 2006). La frecuencia se calculó con el índice de Dajoz (1974) $\mathrm{C}=\mathrm{p}^{*} 100 / \mathrm{P}$, donde $\mathrm{p}$ es el número de muestras donde se encuentra la especie y $\mathrm{P}$ el total de muestras.

\section{RESULTADOS}

\section{Composición faunística}

En el Arco de Scotia, se encontraron representantes de foraminíferos bentónicos de los subórdenes Textulariina $(81,4 \%=61,4$ ejemplares/g), Rotaliina $(17,5 \%=13,3$ ej./g), Miliolina $(0,8 \%=0,64$ ej./g) y Lagenina $(0,3 \%=$ $0,24 \mathrm{ej} . / \mathrm{g})$. Textulariina fue el suborden mejor representado con 9 familias, 11 Géneros y 14 especies. Rotaliina, con menor diversidad brindó

Tabla 3. Número de especies y especies más abundantes según suborden.

\begin{tabular}{lcclc}
\hline \multicolumn{1}{c}{ Suborden } & $\mathrm{N}^{\circ}$ Especies & $\%$ & \multicolumn{1}{c}{$\begin{array}{c}\text { Especies dominantes } \\
\text { dentro del suiborden }\end{array}$} & $\%$ \\
\hline Textularina & 14 & 58,3 & $\begin{array}{l}\text { Miliamina arenácea } \\
\text { Reophax pilulifer }\end{array}$ & 28,4 \\
& & & Trochammina antartica & 16,6 \\
Rotaliina & 6 & 25 & Fursenkoina fusiformis & 15,6 \\
& & & Pullenia subcarinata & 35,5 \\
Lagenina & 2 & 8,3 & Lagenina striata & 25,4 \\
Milionina & 2 & 8,3 & Cornuspira involvens & 83,3 \\
\hline
\end{tabular}


Tabla 4. Especies dominantes en cada muestra

\begin{tabular}{|c|c|c|c|c|c|c|}
\hline Muestra & Especie dominante & $\%$ & $\begin{array}{l}\text { Segunda especie } \\
\text { dominante }\end{array}$ & $\%$ & $\begin{array}{c}\text { Tercera especie } \\
\text { dominante }\end{array}$ & $\%$ \\
\hline $\mathrm{N}^{\circ} 178$ & $\begin{array}{l}\text { Miliammina } \\
\text { arenacea }\end{array}$ & 24,2 & Trochammina inflata & 13,7 & $\begin{array}{l}\text { Ammobaculites } \\
\text { americanus }\end{array}$ & 12,8 \\
\hline $\mathrm{N}^{\circ} 211$ & $\begin{array}{l}\text { Portatrochammina } \\
\text { antartica }\end{array}$ & 27,1 & Trochammina inflata & 20,4 & Bathysiphon sp. & 18,2 \\
\hline $\mathrm{N}^{\circ} 231$ & $\begin{array}{l}\text { Portatrochammina } \\
\text { antartica }\end{array}$ & 18,3 & Miliammina arenacea & 17,2 & Reophax pilulifer & 14,3 \\
\hline$N^{\circ} 243$ & Reophax pilulifer & 36,7 & Bathysiphon sp. & 29,6 & Miliammina arenacea & 23,7 \\
\hline $\mathrm{N}^{\circ} 238$ & $\begin{array}{l}\text { Miliammina } \\
\text { arenacea }\end{array}$ & 54,6 & Reophax pilulifer & 23,6 & Bathysiphon sp. & 8,7 \\
\hline $\mathrm{N}^{\circ} 255$ & $\begin{array}{l}\text { Fursenkoina } \\
\text { fusiformis }\end{array}$ & 34,1 & Angulogerina angulosa & 26,1 & Miliammina arenacea & 12,3 \\
\hline
\end{tabular}

6 familias, 6 géneros y 6 especies. Los subórdenes Miliolina y Lagenina estuvieron pobremente representados con 2 y 1 género, respectivamente y 2 especies cada uno (Tabla 2 ).

\section{Abundancia (N)}

Se recolectó un total de $3.806(76,1$ ejemplares/g) ejemplares. La muestra $\mathrm{N}^{\circ} 231$, obtenida al E de las islas Orcadas, presentó la mayor abundancia (32,1\% de los ejemplares) y la muestra $\mathrm{N}^{\circ} 255$, recolectada al $\mathrm{S}$ de la isla Elefante, la menor abundancia $(6,9 \%$ de los ejemplares). La mayor concentración de foraminíferos se observó en la zona central del área estudiada del Arco de Scotia (Fig. 2). Todas las especies (excepto A. angulosa) tienen su máximo de abundancia en las muestras $N^{\circ} 231$ y $\mathrm{N}^{\circ} 238$ ambas recolectadas en las islas Orcadas (Tabla 5).

Seis especies (25\%) alcanzaron una abundancia relativa superior al 5\%; las especies con mayor abundancia relativa fueron: Miliammina arenacea (23,1\%), Reophax pilulifer $(13,5 \%)$; Portatrochammina antartica $(12,7 \%)$; Bathysiphon sp. (10,7\%); Trochammina inflata $(7,4 \%)$ y Fursenkoina fusiformis $(6,2 \%)$ (Tabla 2$)$.

El desglose de taxa dominantes por muestra pone en evidencia que en la mayoría de las muestras las especies son principalmente foraminíferos de caparazón arenáceo, con excepción de la muestra recolectada en la isla Elefante, donde predominan 2 formas con caparazón calcáreo (Fig. 2). Las especies con mayor representación en cada muestra se presentan en la tabla 4.

Frecuencia

Sólo dos especies, Miliammina arenacea y Pullenia bulloides se encontraron presentes en todas las muestras, mostrando una distribución geográfica amplia, en el Arco de Scotia. Cabe notar que mientras $M$. arenacea aumenta notoriamente el número de ejemplares al Oeste de las Orcadas e isla Elefante, es decir hacia el sur, $P$. bulloides tiende a disminuir en cantidad (Fig. 3A). Labrospira kosterensis, Reophax pilulifer, Bathysiphon sp., Fursenkoina fusiformis y Reophax dentaliniformis se presentaron en 5 de las 6 estaciones estudiadas, estas especies muestran tendencia a concentrarse en las estaciones intermedias (Fig. 3B). Reophax scorpiurus, Pseudobolivina antartica, Cornuspira involvens y Trochammina antartica se recolectaron en 4 de las 6 estaciones, ubicándose preferentemente en el área más septentrional y estando prácticamente ausentes, en las más australes (Fig. 3C). Angulogerina angulosa, Pyrgo sp. y Trochammina inflata se presentaron en 3 de 6 (Fig. 3D). Lagena striata, Cassidulinoides parvus, Cyclammina cancellata, Trochammina malovensis, Bulimina aculeata, Lagena distoma y Buccella frigida se presentaron en 2 de 6 estaciones. Cabe destacar que 5 de estas especies son calcáreas y su mayor abundancia se observó en las estaciones de más 

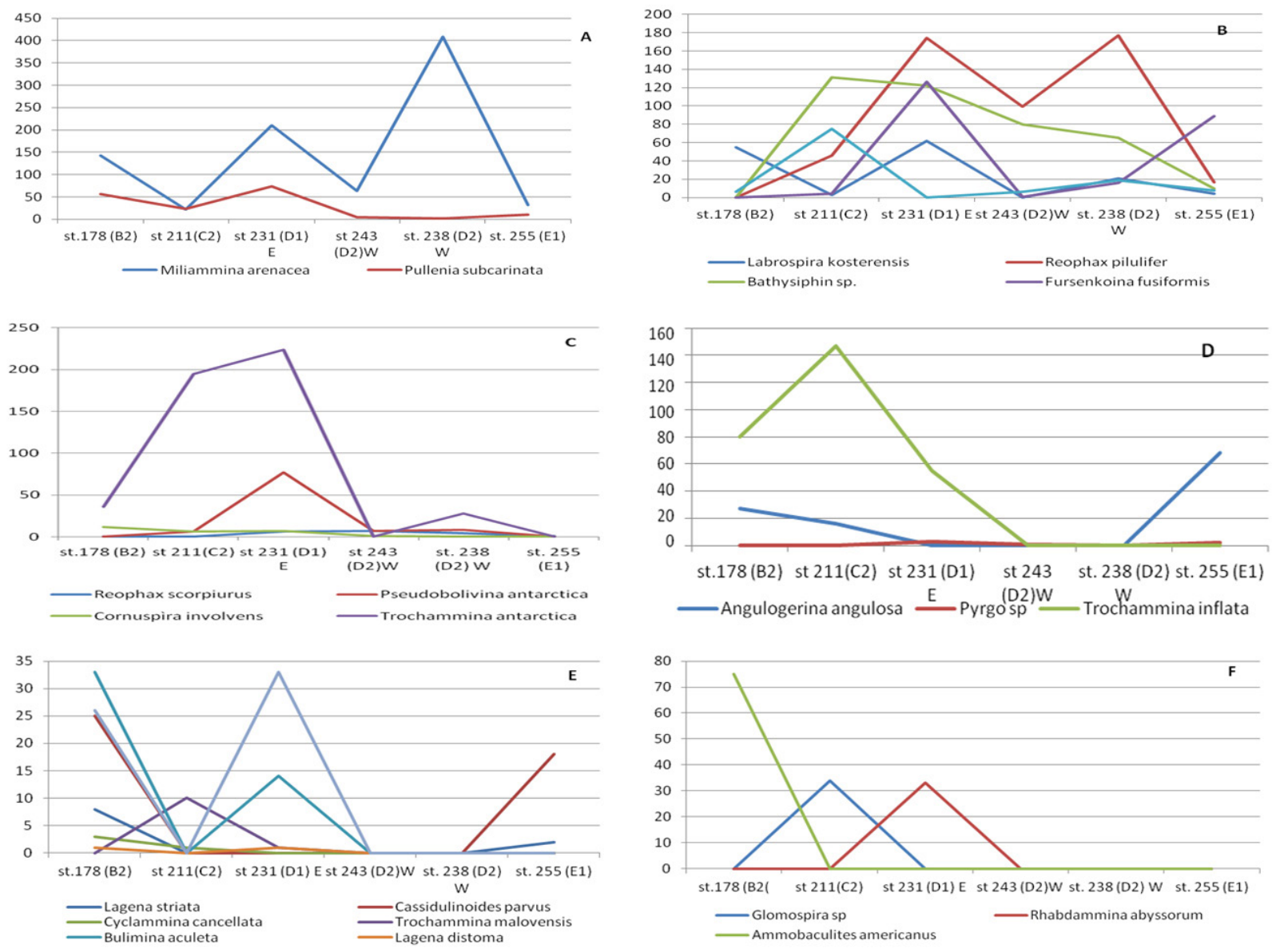

Fig. 3. Distribución de las especies en la muestras; (A) especies presentes en todas las muestras; (B), especies presentes en 5 de las 6 muestras; (C), especies presentes en 4 de las 6 muestras; (D) especies presentes en la mitad de las muestras; (E), especies presentes en 2 de las 6 muestras y (F) especies presentes en una sola muestra.

al norte. (Fig. 3E). Finalmente, A. americanus, $R$. abyssorum y Glomospira sp. se presentaron solo en una muestra y en las localidades más cercanas al norte: Georgias del Sur, Sandwich y al E de las islas Orcadas (Fig. 3E). En estos dos últimos grupos además de presentarse en un número mínimo de muestras, aportaron con escasos ejemplares.

\section{Riqueza específica (S)}

Se recolectó un total de 24 especies. El menor número de especies (10 especies) se obtuvo en las muestras $\mathrm{N}^{\circ} 243$ y 238 , recolectadas en SW de las islas Orcadas, y el mayor (17 especies) en la muestra $\mathrm{N}^{\circ} 231$, recolectada en el $\mathrm{E}$ de las islas Orcadas. Se observó una disminución de especies hacia el sur (Tabla 5).

\section{Equidad (J')}

El índice osciló entre 0,597 y 0,849, mostrando una tendencia aunque no tan clara de dominancia de algunos taxa. En la muestra $\mathrm{N}^{\circ}$ 238 se registró el valor más bajo $(0,597)$, lo que indicaría una mayor dominancia. Considerando las 3 especies con mayor representación, en la muestra $\mathrm{N}^{\circ} 178$, conformaron el $50,7 \%$ de los ejemplares; en la $\mathrm{N}^{\circ} 211$, el $65,7 \%$; en la $\mathrm{N}^{\circ}$ 231, el 49,8\%; en la $\mathrm{N}^{\circ} 243$, el $90 \%$; en la $\mathrm{N}^{\circ}$ 238, el 86,9\% y en la $\mathrm{N}^{\circ} 255$ el 72,5\%. Este parámetro no presentó gran variación entre las 
Tabla 5. Parámetros ecológicos univariados

\begin{tabular}{lccccc}
\hline Muestra & $\begin{array}{c}\text { Número de } \\
\text { especies (S) }\end{array}$ & $\begin{array}{c}\text { Número } \\
\text { ejemplares (N) }\end{array}$ & Densidad (ej./g) & Equidad (J') & $\begin{array}{c}\text { Diversidad } \\
\text { H'(loge) }\end{array}$ \\
\hline 178 (B2) & 15 & 586 & 11,72 & 0,849 & 2,298 \\
211 (C2) & 15 & 719 & 14,38 & 0,756 & 2,046 \\
231 (D1) & 17 & 1221 & 24,42 & 0,818 & 2,318 \\
243 (D2) & 10 & 270 & 5,4 & 0,638 & 1,468 \\
238 (D2) & 10 & 749 & 14,98 & 0,597 & 1,374 \\
255 (E1) & 12 & 261 & 5,22 & 0,746 & 1,854 \\
\hline
\end{tabular}

distintas muestras (Tabla 5).

Diversidad (H')

De las seis muestras analizadas, en tres el indice de Shannon-Wiener fue $>$ de 2, y correspondió a las muestras recolectadas en las localidades de más al norte (muestras $\mathrm{N}^{\circ} 178, \mathrm{~N}^{\circ} 211$ y No 231) del Arco, valores sobre 2 indicando una diversidad normal y tres, (situadas más cercanas a Antártica), brindaron valores bajo 2 , indicando una tendencia a la dominancia de alguna especie (Tabla 5).

En relación a la presencia de formas con caparazón calcáreo versus especies con caparazón arenáceo se observó una mayor cantidad de especies con caparazón arenáceo (58,3\%) (Fig. 4 A) y en relación al número de ejemplares, los foraminíferos con caparazón arenáceo triplicaron la cantidad de ejemplares con caparazón calcáreo (81,3\%) (Fig.4 B).

\section{Análisis de conglomerados}

El análisis de conglomerados revela que la mayor afinidad se encuentra entre las muestras $\mathrm{N}^{\circ} 243$ y $\mathrm{N}^{\circ} 238$ ambas situadas en las islas Orcadas, y entre las muestras $\mathrm{N}^{\circ} 211$ y $\mathrm{N}^{\circ} 231$ (islas Sandwich e islas Orcadas), con las que forman un grupo mayor. La mayor disimilitud se observa entre la muestras $N^{\circ} 178$ en el extremo norte del arco de Scotia y N ${ }^{\circ} 255$ en el extremo sur del Arco (isla Elefante) (Fig. 5).

\section{Comparación con áreas \\ subantártica y antártica}

La revisión bibliográfica permitió detectar que
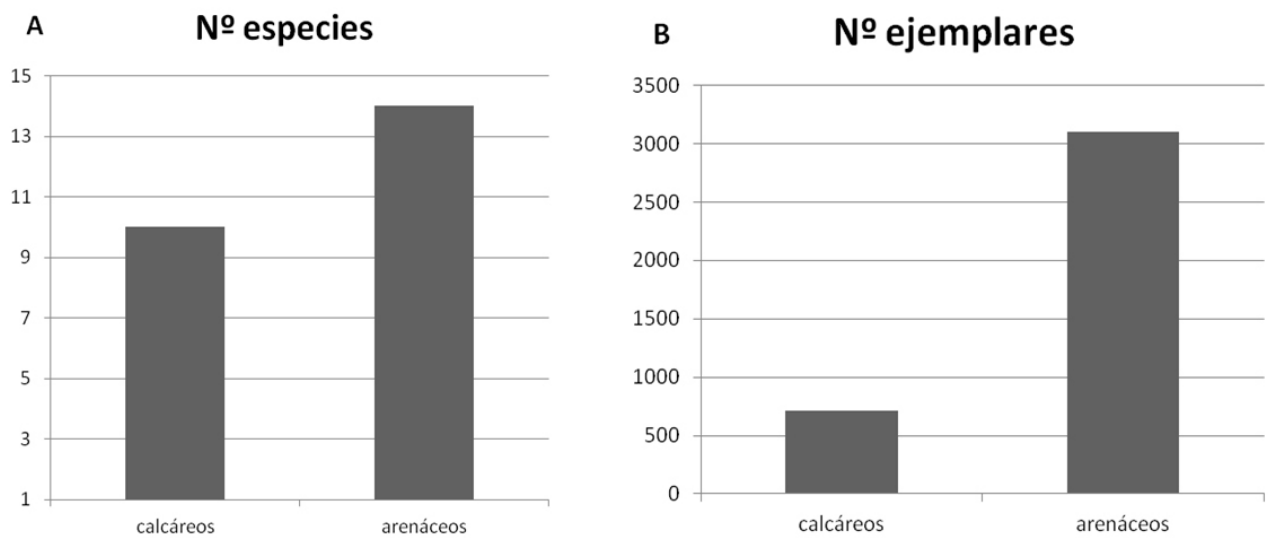

Fig. 4. Relación del número de especies con capazón calcáreos versus arenáceos (A) y relación de número de ejemplares con caparazón calcáreo versus arenáceos (B) 


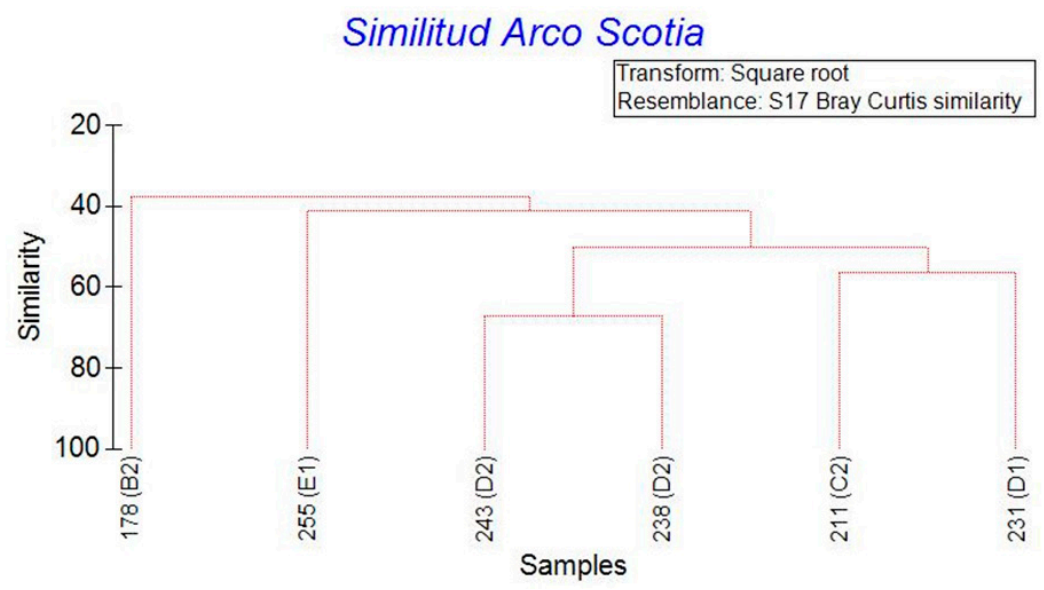

Fig. 5. Análisis de conglomerados.

la mayoría de las especies recolectadas en el Arco de Scotia están también presentes en Antártica y Pacífico, aunque en cantidades inferiores. Las siguientes especies recolectadas en el Arco de Scotia han sido registradas tanto en el pacífico como en Antártica: Angulogerina angulosa; Bucella frígida; Bulimina aculeata; Cassidulinoides parvus; Cornuspira involvens; Lagena distoma; Lagena striata; Miliammina arenacea; Pullenia subcarinata; Reophax dentaliniformes; Reophax pilulifer; Reophax scorpiurus y Fursenkoina fusiformis, lo que permite inferir que se trasladan de uno a otro continente usando el sistema de islas del Arco de Scotia. Solo unas pocas como Ammobaculites americanus (2\%) y Cyclammina cancellata $(0,1 \%)$ presentes en el Pacífico y en el Arco de Scotia, no se mencionan en Antártica, lo que sugiere que se encuentran restringidas al área Pacífica, presentándose en el Arco de Scotia en abundancias bajas. Por otro lado, Pseudobolivina antarctica; Portatrochammina antartica y Trochammina malovensis se han recolectado solo en la Antártica (datos tomados de Lena (1975, en Isla Elefante); Lena (1980, en Noroeste península antártica); Finger \& Lipps (1981, en Isla Decepción); Mateu (1989 en península Antártica); Martínez \& Martínez (1994 en bahía Chile); Hromic ${ }^{1}$ (1997 en Isla Decepción); Hromic \& Páez² (1999 bahía Chile).

\footnotetext{
${ }^{1}$ Hromic, M. T., 1997."Foraminíferos antárticos recientes y sus vinculaciones con la microfauna sudamericana". Informe final presentado al INACH. Informe Instituto de la Patagonia. Universidad de Magallanes, Punta Arenas, Chile.
}

Majewski (2005) señala que algunos autores ponen en sinonimia $P$. antartica y $T$. malovensis, en este trabajo se han considerado especies distintas.

\section{DISCUSIÓN}

Un inusual elevado número de foraminíferos bentónicos con caparazón arenáceo se hizo presente en las muestras ( $81,3 \%$ de los ejemplares), lo que difiere completamente de lo encontrado en la costa pacífica, donde los arenáceos llegan solo al $10 \%$ y de la Antártica, donde alcanzan el $48 \%$ (Hromic 2012), este último dato es semejante a lo que sucede en ambientes periglaciales donde los foraminíferos arenáceos alcanzan el 45,8\% (Hromic \& Montiel 2011). En la isla Elefante el porcentaje de foraminíferos con caparazón arenáceo se vio muy disminuido (27,6\%), lo que concuerda con las observaciones de Lena (1975). Hromic (2011b) señala que entre los $52^{\circ}-56^{\circ} \mathrm{S}$, en el territorio austral chileno, solo un $2 \%$ de los foraminíferos son arenáceos. Su número aumentaría en canales y fiordos interiores en donde llegarían hasta el $18 \%$ (Hromic, 2011a).

En relación a las especies dominantes dentro de este grupo, Miliammina arenacea posee hábitos infaunales (Haynes, 1981; Murray, 1991), y Portatrochammina antartica es epifaunal,

${ }^{2}$ Hromic, T. \& M. Páez 1999. Asociación de foraminíferos recientes en bahía Chile (62 ${ }^{\circ} 28^{\prime}$ s; $\left.59^{\circ} 41^{\prime} w\right)$, isla Greenwich, Shetland del Sur, Antártica. Expedición ECA XXXII, INACH. Congreso Arctic and Antartic, Poles apart? Universidad de Magallanes, Punta Arenas, Chile 
intersticial o se adhiere al sustrato (Kitazato 1984), formas de vida que se acomodarían a la presencia por una parte de sustratos duros, de origen volcánico abundantes en el Arco de Scotia y por otro, a la fuerte Corriente Circumpolar del sector (Arntz \& Brey, 2003). M. arenacea tiene alta representación y está ampliamente distribuida en Antártica (Kennet, 1967; Osterman \& Kellog, 1979, Lena, 1980, etc.). Según Schroeder-Adams (1990) es endémica de Antártica lo que sugiere que avanzan desde el sur hacia Sudamérica. La escasa presencia de Miliamina antartica en las costa Pacífica y su restricción a zonas periglaciales (Zúñiga-Rival, 2006) y su ausencia total en Isla de los Estados (Thompson, 1978) sugiere que esta especie no se adapta bien a ambientes probablemente más temperados. $P$. antartica también sería endémica y de amplia distribución en Antártica que tampoco se ha podido adaptar a latitudes más bajas. Los reofácidos (R. pilulifer, $R$. scorpiurus y $R$. dentalinifomes) son de hábitos infaunal, pero se establecen más profundamente en los sedimentos, lo que les permitiría resistir condiciones más adversas. $R$. pilulifer, aunque con escasos ejemplares suele encontrase en Sudamérica. A. angulosa y Bucella frígida son típicos representantes de canales y fiordos patagónicos, pero mientras $A$. angulosa prefiere ambientes más oceánicos y profundos, $B$. frigida se le encuentra con frecuencia en ambientes someros (Hromic, 2011 y b, 2012; Hromic et al. 2006; Zapata \& Moyano, 1997). Los lagénidos (Lagena striata y L. distoma) son cosmopolitas, frecuentes en el Pacífico aunque con escasa representación. B. aculeata y $P$. subcarinata también son cosmopolitas, de lo que se desprende que se adaptan bien a distintos ambientes (Loeblich \& Tappan, 1988)

Earland (1934), encontró un predominio de fauna arenácea tanto en número de ejemplares como de especies, escasos calcáreos Miliólidos y de gran tamaño como algunos Pyrgo. Entre las especies más abundantes Earland menciona: Astrorhyza polygona, Pilulina jefreysii (=Labrospira), Thurammina spumosa, T. corrugata, Bathysiphon argillaceus $y$ Ehrenbergina parva (= Cassidulinoides parvus). Este autor no registró $M$. arenacea ni $P$. antartica, tan abundantes en el área, pero como se mencionó anteriormente ello podría deberse a las técnicas de muestreo y a la profundidad desde donde se extrajeron las muestras.

Respecto de la riqueza de especies, en el Arco de Scotia el número de especies fue bastante menor, (10 a 17 especies/muestra), que en los sectores continentales. Hromic (2011b) recolectó un total de 170 especies entre $52^{\circ}-56^{\circ} \mathrm{S}$, que fluctuaron entre 5 y 42 especies/ muestra. Más al norte, entre $47^{\circ}-50^{\circ} \mathrm{S}$ se identificaron 88 especies, (con rango entre 3 y 42 especies/muestra) (Hromic, 2011a). En Antártica, Earland (1934), analizó una cincuentena de muestras procedentes del área del estrecho Bransfield e Islas Shetland del Sur, encontrando no más de 50 especies totales con un promedio de 30 especies por muestra. Estas diferencias podrían vincularse al sedimento escaso del sector y a las fuertes corrientes, que tienden a lavar el sustrato (Arntz \& Brey, 2003).

Earland (op cit.) también puso en evidencia la gran influencia del pacífico, puesto que encontró representantes de: Bolivina compacta, Bulimina patagonica, Cassidulina pulchella, Cassidulina laevigata, Cibicides dispars, Dendronina arborescens var. antarctica, Discorbis turbo, Discorbis vilardeboana, Ehrenbergina pupa, Elphidium macellum, Elphidium owenianum, Globigerina bulloides, Globigerina triloba, Globorotalia crassa, Globorotalia manardii, Hyperammina clavigera, Lagena alveolata var. separans, Lagena quadralata, Lagena revertens, Milioina insignis, Nonion sloanii, Opthalmidium margaritiformis, Rotalia beccarii (=Ammonia), Virgulina schreibersiana var. complanata. Sin embargo ninguna de estas especies fue encontrada en el Arco de Scotia, lo que se relaciona con la profundidad de las muestras.

En relación a la frecuencia, se evidencia la adaptabilidad de $M$. arenacea y de Pullenia subcarinata ya que ambas especies aparecen en todas las muestras. Sin embargo, dado el hecho de que la abundancia de $M$. arenacea tiende a disminuir hacia el norte y por el contrario, la de $P$. subcarinata lo haga hacia el sur, podría asociarse al desplazamiento de la Convergencia Antártica (Arntz \& Brey, 2003) lo que permitiría que $M$. arenácea, cuyo centro de origen sería Antártica, se encuentre más al norte del continente y por el contrario para $P$. subcarinata el centro de 
origen sería Sudamérica y se desplazaría más al sur de este continente, llegando sin dificultad a Antártica donde es frecuente en la península, al menos sus caparazones (Mayewski, 2005).

\section{CONCLUSIONES}

En el Arco de Scotia se recolectaron 24 especies de foraminíferos bentónicos las que se clasificaron en 4 subórdenes, 18 familias y 20 géneros. Textulariina, (foraminíferos con caparazón arenáceo), dominó tanto en abundancia como riqueza de especies, y su abundancia relativa fue muy superior a la registrada en Sudamérica y Antártica.

Las especies más abundantes fueron: Miliammina arenacea (23,1\%), Reophax pilulifer (13,5\%); Portatrochammina antartica (12,7\%); Bathysiphon sp. (10,7\%); Trochammina inflata $(7,4 \%)$ y Fursenkoina fusiformis $(6,2 \%)$.

Respecto de la riqueza de especies, en el Arco de Scotia fue bastante menor, (10 a 17 especies / muestra), que en los sectores continentales. Tanto la diversidad (indice de Shannon-Wiener) como la equidad (ídice de Pielou) indican que no hay dominacia de especies.

El análisis de conglomerados mostró que las estaciones extremas (SE Georgias del Sur e Isla Elefante), son más disímiles en su composición faunística.

Finalmente, se concluye que el Arco de Scotia actúa como corredor biológico para especies como: Angulogerina angulosa; Bucella frígida; Bulimina aculeata; Cassidulinoides parvus; Cornuspira involvens; Lagena distoma; Lagena striata; Miliammina arenacea; Pullenia subcarinata; Reophax dentaliniformes; Reophax pilulifer; Reophax scorpiurus y Fursenkoina fusiformis. Especies como Ammobaculites americanus y Cyclammina cancellata, propias de Sudamérica y Portatrochammina antartica, Pseudobolivina antartica y Trochammina malovensis, endémicas de Antartica el Arco de Scotia marcaría su límite biogeográfico.

\section{AGRADECIMIENTOS}

La autora agradece al Instituto Alfred Wegener por permitirle usar las muestras. A la Universidad de Magallanes e Instituto de la Patagonia, por las facilidades para realizar esta investigación, en especial al Dr. Carlos Ríos por recolectar las muestras y al Dr. Américo Montiel por su ayuda con el análisis estadístico. A la Srta. Lyta Quezada, por su valiosa cooperación en la preparación de las muestras.

\section{LITERATURA CITADA}

Adl, S. M., Simpson, A. G. B., Farmer, M. A., Andersen, R. A., Anderson, O. R., Barta, J. R., Bowser, S. S., Brugerolle, G., Fensome, R. A., Fredericq, S., James, T. Y., Karpov, S., Kugrens, P., Krug, J., Lane, C. E., Lewis, L. A., Lodge, J., Lynn, D. H., Mann, D. G., Mccourt, R. M., Mendoza, L., Moestrup, ø., Mozley-Standridge, S. E., Nerad, T. A., Shearer, C. A., Smirnov, A. V., Spiegel, F. W. \& Taylor, M. F. J. R. (2005). The New Higher Level Classification of Eukaryotes with Emphasis on the Taxonomy of Protists. Journal of Eukaryotic Microbiology, 52(5), 399-451.

Arntz, W. \& A. Brey (Eds.), 2003. Expedition ANTARKTIS XIX/5 (LAMPOS) of RV. "Polarstern" in 2002 Be. Ber. Polarforsch. Meeresforsch.

Barker, R. W. (1960). Taxonomic Notes on the Species figured by H. B. Brady in his Report on the Foraminifera dredged by H.M. S. Challenger during the years 18731876. Society Economical, Paleontological \& Mineral. Special Pub. N ${ }^{\circ}$ 9, Oklahoma USA.

Boltovskoy, E. (1965). Foraminiferos recientes. Eudeba. Buenos Aires.

Boltovskoy, E. G. Giussani, S. Watanabe \& R. Wright (1980). Atlas of benthic shelf foraminifera of the southwest Atlantic Junk by Pub. The Hague-Boston-London.

Brady, H. B. (1884). Report on the Foraminifera dredged by H.M.S. "Challenger" during the years 1873 -1876. Report Voyage Challenger, Zoology, 9,1-814.

Clarke, K. \& R. Gorley. (2006). Primer v.6 User Manual/Tutorial PRIMER-E Ltd.: Plymouth Marine Laboratory. UK.

Dajoz, R. (1974) Tratado de Ecología. MundiPrensa, Madrid. 
Decrouez, D. (1989). Generic ranges of Foraminiferida. Revue Paleobiologie, 8(1), 263-321.

Earland, A. (1934). Foraminifera Part III. The Falklands sector off the Antartic (excluding South Georgia). Discovery Rep. X, 1-208. pl. I-X.

Ellis, B. \& A. Messina. (1940) (et seq.). Catalogue of Foraminifera. American Museum of Natural History. Special Pub. 30 volúmenes.

Finger L. \& J. Lipps (1981). Foraminiferal decimation and repopulation in an active volcanic caldera, Deception Island, Antarctica. Micropaleontology, 27(2), 11139.

Gaździcki, A. \& W. Majewski (2003). Recent foraminifera from Goulden Cove of King George Island, Antarctica. Polish Polar Research, 24(1), 3-12.

Haynes J. R. (1981). Foraminifera. Macmillan, N. $\mathrm{Y}$.

Herb, R. (1971). Distribution of recent benthonic foraminifera in the Drake Passage. Antarctic Research, 17, 251-300.

Heron- Allen, E. \& A. Earland (1932). Foraminifera. Pt.1. The ice-free area of the Falkland Islands and adjacent watter. Discovery Rep., 4, 291460.

Hromic, T. (1996). Foraminíferos bentónicos (Protozoa: Foraminiferida) de aguas profundas del estrecho de Magallanes, Chile. Anales Instituto Patagonia, Ser. Cs. Nats. (Chile), 24, 65-86

Hromic, T. (1999). Foraminíferos bentónicos de canales australes de Kirke: canal Kirke, golfo Almte. Montt y seno Última Esperanza, XII ${ }^{\mathrm{a}}$ Región, Magallanes y Antártica chilena. Anales Instituto Patagonia Ser. Cs. Nat. (Chile), 27, 91-104.

Hromic, T. (2001). Foraminíferos bentónicos del canal Baker $\left(47^{\circ} \mathrm{S} ; 7^{\circ} \mathrm{W}\right)$ Pacífico sudoriental, Chile. Anales Instituto Patagonia Ser. Cs. Nat., 29,135-156.

Hromic, T. (2002). Foraminíferos bentónicos de bahía Nassau, Cabo de Hornos, Chile. Comparación con foraminíferos del cono sur de América, Antártica y Malvinas. Anales Instituto Patagonia Ser. Cs. Nat., 30, 95-108.
Hromic, T. (2005). Foraminíferos bentónicos entre seno Reloncaví y Golfo Corcovado. Crucero Cimar 10 Fiordos, Informes preliminares: $125-134$.

Hromic, T. (2007). Biodiversidad y ecología del microbentos (Foraminifera: Protozoa) entre la boca del Guafo y golfo de Penas (43$\left.46^{\circ} \mathrm{S}\right)$, Chile. Ciencia y Tecnología del Mar, 30(1), 89-103.

Hromic, T. (2009). Distribución batimétrica de foraminíferos bentónicos (Protozoa: Foraminiferida) al sur del estrecho de Magallanes $\left(52^{\circ}-56^{\circ} \mathrm{S}\right)$, Chile. Anales Instituto Patagonia, Chile, 37(1), 23-38.

Hromic, T. (2011a). Foraminíferos bentónicos recolectados durante la expedición Cimar 14 Fiordos, Patagonia Chilena. Anales Instituto Patagonia (Chile), 39(1), 75-89.

Hromic, T. (2011b). Foraminíferos bentónicos recientes del Estrecho de Magallanes y canales australes chilenos Cimar 3 Fiordos $\left(52^{\circ}-56^{\circ} \mathrm{S}\right)$ Anales Instituto Patagonia (Chile) 39(2), 17-32.

Hromic, T. (2012). Foraminíferos bentónicos de la expedición Cimar 11 Fiordos, canales patagónicos chilenos: Biodiversidad $y$ abundancia. Ciencia y Tecnología del Mar, 35, 49-70.

Hromic, T. \& A. Montiel (2011). Foraminíferos bentónicos de seno Gallegos y bahía Brookes $\left(54,5^{\circ} \mathrm{S}-69,5^{\circ} \mathrm{S}\right)$, Chile: Patrones de distribución y diversidad). Anales Instituto Patagonia, 39(2), 33-46

Hromic, T. M. Camblor \& L. Quezada (2012). Foraminíferos textuláridos en sedimentos someros recientes de la península antártica y su relación con el área subantártica sudamericana. Anales Instituto Patagonia (Chile), 40(1), 125-138

Hromic, T., S. Ishman \& N. Silva (2006). Benthic foraminiferal distribution in Chilean fjords: $47^{\circ} \mathrm{S}$ to $54^{\circ} \mathrm{S}$ Marine Micropaleontology, 59, 115-134.

Ishman, S. \& E. Domack (1994). Oceanographic controls on benthic foraminifers from the Bellingshausen margin of the Antarctic Peninsula. Marine Micropaleontology 24, 119-155.

Ishman, S. \& R. Martínez (1995). Distribution of 
modern Benthic foraminifers from fjord región of southern Chile $\left(42^{\circ} \mathrm{S}\right.$ to $\left.55^{\circ} \mathrm{S}\right)$. Antarctic Journal Review. 6-8.

Kennett J. P. (1967). New foraminifera from the Ross Sea, Antarctica. Contributions from the Cushman Foundation for Foraminiferal Research, 18(3), 133-135.

Kitazato, H. (1984). Microhabitats of benthic foraminifera and their application to fossil assemblages. Benthos, 83, 339- 344,

Lena, H. (1975). Foraminíferos bentónicos del área de isla Elefante (Antartida). Physis, Secc. A, 34(89), 405-431.

Lena, H. (1980). Foraminíferos bentónicos del noroeste de la Península Antártica Physis, Secc. A, 39(96), 9-20.

Loeblich, A. \& H. Tappan (1988). Foraminiferal Genera and Their Classifications. Van Nostrand Reinhold Co. N.Y. Text - vol: 970. Pl - Vol $212 p+847$.

Mackensen, A. H. Grobe, G. Kuhn \& D. K. Füterer (1990). Benthic foraminiferal assemblages from the eastern Weddell sea Between 68 and $73^{\circ} \mathrm{S}$ : Distribution, ecology and fossilization potential. Marine Micropaleontology 16, 241-283.

Magurran, A. E. (1988). Ecological diversity and its mesasurement. Princeton University Press. New Jersey.

Majewski, W. (2005). Benthic foraminiferal communities: distribution and ecology in Admiralty Bay, King George Island, West Antarctica. Polish Polar Research, 26(3), 159-214.

Marchant, M. (1993). Foraminíferos de la Bahía Scholl, Región Magallánica, Chile (Protozoa: Foraminifera) Gayana Zoology, 57(1), 6175.

Martínez, G.R. \&R. Martínez(1994). Foraminíferos bentónicos recientes de Bahía Chile, Isla Greenwich, Islas Shetland del Sur. Su significado para la historia Paleoclimática Holocena de la Antártica Occidental. $7^{\circ}$ Congreso Geológico Chileno. Universidad de Concepción, Chile: 1689-1692. Actas

Mateu, G. (1989). Micropaleontología sedimentaria. Informe de resultados de la campaña "Antartida 8611". Pub. Esp. del Inst. Español de Oceanografía, $1^{\mathrm{a}}$ Exp. Cient. Pesquera en la Antartida 1986-1987. Sec. Gral. de Pesca Marítima M.A.P.A. Min. Agric., Pesca y Alim. Madrid, 2, 83-173.

Murray, J. W. (1991). Ecology and paleoecology of benthic foraminifera. Longman Scientific \& Technical. Avon.

Osterman, L. E. \& T. B. Kellogg (1979). Recent benthic foraminiferal distribution from the Ross Sea, Antarctica: relation to ecologic and oceanographic conditions. Journal of Foraminiferal Research, 9(3), 250-269.

Schroeder-Adam, C. (1990). High latitudes agglutinated foraminífera. Prydz Bay (Antartica) VS Lancaster Sound Canadian Arctic. NATO. ASI. C, 327, 315-343.

Thompson, L. (1978). Distribution of living benthic foraminifera, Isla de los Estados, Tierra del Fuego, Argentina. Journal Foraminiferal Research, 8(3), 241-257.

Violanti, D., B. Loi \& R. Melis (2000). Distribution of Recent Foraminifera from the Strait of Magellan. First quantitative data. Bolletino Museo Regionali Scientia Naturalli, Torino, 17(2), 511-539.

Zapata, J. \& H. Moyano (1997). Foraminíferos bentónicos recientes de Chile Austral. Boletín de la Sociedad Biología, Concepción, Chile, 68, 27-37.

Zúñiga-Rival, M. (2006). Estudio preliminar de los foraminíferos bentónicos (Protozoa: Foraminiferida) de bahía Yendegaia, Tierra del Fuego, Chile. Anales Instituto Patagonia (Chile), 34, 33-40. 
